\title{
Intelligent Construction and Flexible Construction
}

\author{
Chengshui YU ${ }^{\text {a, } 1}$ \\ ${ }^{a}$ CRCC Development Group Co.,LTD, Beijing 100041, China
}

\begin{abstract}
Today, in the digital era, all walks of life have diversified development under the penetration of information technology, and the traditional construction industry is inevitably combined with information technology to move toward the direction of intelligent construction. As a broad concept, intelligent construction includes many theories and multiple directions of research, from design to construction to post-maintenance, while flexible construction focuses on the organization of production mode. In this paper, through the comparative analysis of intelligent construction and flexible construction, the framework of intelligent construction and flexible construction is established to deepen the understanding of the two modes, so as to correctly control the direction of intelligent development, and provide a basis for the construction mode of the intelligent era.
\end{abstract}

Keywords. Intelligent construction, flexible construction, digital, artificial intelligence

\section{Introduction}

With the development of information theory and computer technology, information technology has penetrated into all walks of life, and the management and analysis of large amounts of data has become the basic means of various research. Building construction, as a project that integrates a variety of specialties, has been a very complicated affair since its appearance. Nowadays, the construction industry still continues the labor-intensive organization mechanism. Under the digital background of the information age, intelligent construction offers a glimpse into the problem.

\section{Basic Theory of Intelligent Construction}

\subsection{Intelligent Construction}

Intelligent construction is a new construction mode, which integrates intelligence, construction process and informatization. The current intelligent construction includes BIM (building information model) technology, artificial intelligence technology, 3d printing technology, and big data internet of things technology. The generation of intelligent construction simplifies the tedious construction process in the traditional construction method, integrates the information of various specialties, saves labor cost,

1 Chengshui Yu, CRCC Development Group Co., LTD, Beijing 100041, China; E-mail: yuchengshui000@163.com. 
reduces construction costs, and accurately constructs the construction technology. Intelligent construction is a new round of revolution in the construction industry. In the intelligent construction, the robot technology combined with artificial intelligence is used to replace the traditional construction method, and the design process modeling and simulation of the building are intelligentized. At the same time, the big data internet of things technology is used for construction management in the construction process. Intelligent construction is becoming the trend of construction industry, and plays an increasingly important role in construction projects.

\subsection{Main Technical Parameter}

\subsubsection{Building Information Modeling (BIM) Technology}

BIM technology is developed by Autodesk company, which provides auxiliary design for the construction industry, including all the information from the beginning of architectural design, medium-term construction, and later maintenance and operation. It is integrated into a three-dimensional model, which provides an information exchange and sharing platform for engineers of different majors. Engineers can work collaboratively according to BIM technology, which not only improves work efficiency, but also saves resources and reduces construction costs. BIM technology has developed early in europe and the united states and other countries, and the technology is relatively mature. China' s development time is late, but the development is relatively rapid. Most construction-related enterprises have begun to study and apply BIM technology [1].

\subsubsection{Intelligent Robot}

At present, the application of intelligent robots in the construction industry is common in industrial robots, namely mechanical arm system. Mechanical arm as a widely used equipment in manufacturing industry has been quite complete tool. By programming, the manipulator can be controlled to construct large components, which makes architects came to know the mechanical arm. The mechanical arm can complete the prefabricated construction in large-scale processing, and the highly free programming control enables the manipulator to build various complex shapes. Today, with the development of nonlinear architecture and customization, the mechanical arm can combine various materials for personalized construction. At present, many universities and research institutions have studied the application of mechanical arm in intelligent construction. Research teams at Zurich university of technology and Helsinki alto university, for example, built a large brick maze in the laboratory using robotic arms by pinpointing the location of each brick and programming the building sequence (figure 1) [2]. Research teams at the university of science and technology in Singapore have conducted research on digitally controlled shotcrete construction using robotic arms (figure 2) [3]. 


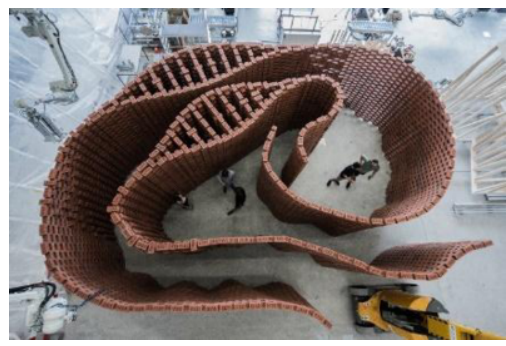

Figure 1. The brick labyrinth in the robotic fabrication laboratory at eth Zurich, 2017.

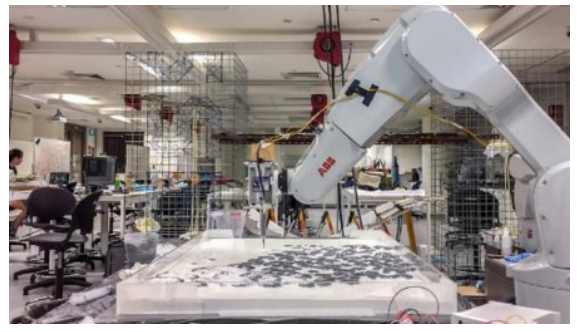

Figure 2. The mechanical arm controls the piston to inject liquid aluminium.

\subsubsection{Internet of Things Technology}

The internet of things is an important part of the new generation of information technology. The idea of connecting things is very suitable for building construction, a complex system involving many realistic objects, such as coding materials and components to achieve the purpose of intelligent management [4]. At present, in the construction industry, the application of the internet of things is mainly reflected in the construction management and the improvement of the environment of managers [5]. China has applied the internet of things technology in the construction industry in 2012, which realizes the information sharing among the prefabricated components, construction personnel and management personnel of the building. Through the coding processing of the prefabricated components of the building, the prefabricated components can be accurately positioned. When problems are found in the construction, they can be accurately positioned to the location of the problem for rapid solution, avoiding a series of economic losses. At present, the application of the internet of things in the construction industry in China has achieved initial success.

\subsubsection{D Printing Technology}

3D printing has the characteristics of integrated molding and convenient construction, and its printing materials are also extended to concrete, metal, plastic, sawdust and other building materials. This makes housing construction simpler, and this technology also enables complex buildings with nonlinear forms to be constructed conveniently and accurately. This different from the traditional mode of production of building production relations were reconstructed. This construction technology first by $3 \mathrm{~d}$ printing technology in China is also booming, has been useful $3 \mathrm{~d}$ printing technology to build housing mature cases. Professor $\mathrm{Xu}$ Weiguo team of Tsinghua university school of architecture has independently developed 'robot $3 \mathrm{~d}$ printing concrete mobile platform' and 'rapid construction system of concrete buildings'. Recently, these two scientific and technological achievements have been successfully applied to the actual printing construction of model houses in low-income housing in Africa (figure 3) [6]. 


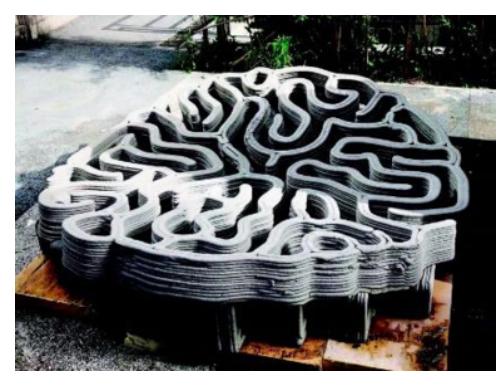

Figure 3. 3D printing of concrete using a robotic arm

\section{Flexible Construction}

\subsection{Concepts Related to Flexible Construction}

With the penetration of information technology into all walks of life, the construction industry inevitably moves towards the road of digitalization. The continuous progress of digital design tools and matching production and processing tools promotes the progress of construction quality. The emergence of various architectural forms such as parametricism and nonlinearity also puts forward new requirements for construction. At present, the construction industry still continues the labor-intensive organization mechanism, the production management mode is old, the technology of the information age is not effectively absorbed, the construction efficiency is low, the professional cooperation is not close, the waste of various resources is serious, the safety accidents occur frequently, the engineering quality is seriously affected by the technical level of different construction personnel, and the requirements of lean construction are not satisfied. With the fourth industrial revolution and the proposal of ' China construction $2035^{\prime}$ strategy, the traditional construction industry is undergoing transformation and upgrading in the information age. The trend of intelligent construction is inevitable. Information technology represented by big data and artificial intelligence is being widely studied how to apply to the field of construction. Getting rid of the current construction dilemma and achieving the goal of lean construction have also become an urgent problem in the development of intelligent construction.

Flexible construction is a construction mode that accurately controls all aspects of construction and production through the comprehensive application of various technologies in the intelligent era, and makes full use of digital technology to achieve flexible design, construction, management, docking and operation. The flexibility in the flexible construction can be reflected in two parts: 1) the building needs to adapt to the continuous change of external causes in the construction process, whether the construction process of the building can meet the requirements of the outside world at any time. 2) whether the building can adapt to the internal changes in the construction process, for example, in the construction process if there is a case of construction machinery damage, construction efficiency and completion and in the absence of construction machinery damage in the case of construction efficiency and completion of the difference, if there is a difference, the ratio of the two cases to measure. The traditional construction method can be defined as "rigidity". The advantage of this construction method is that it can realize the mass production of a building component 
with high production efficiency. Since the precast concrete components of the same housing space in the construction process are the same, the utilization rate of the equipment for pouring precast concrete is high and the price of the single piece is low. However, this "rigidity" construction has several shortcomings compared with the "flexible" construction, namely, it is difficult to cope with the production of products with diverse types and small demand. If the enterprise masters the flexible construction, the construction cost is low, and can be diversified construction. At present, the construction market is gradually moving towards dynamic construction. Flexible construction system can not only reduce costs, but also enrich construction types and improve construction quality, which is the main competitive conditions in the construction market.

\subsection{Characteristics of Flexible Construction}

Flexible construction mainly includes the following characteristics:

Mechanical flexibility used in construction: when investors need to build a number of different types of buildings at the same time, the mechanical part can process different parts with the change of construction products.

- Process flexibility in construction: when the process of construction is constant, the building itself can adapt to the change of raw materials. When the raw materials change during construction, the construction system can be corresponding to the corresponding construction system according to the change of raw materials.

- Product flexibility: when the appearance, function, layout and other elements of the building are changing with the times, the flexible system can be very economical and rapid to keep up with the pace of the times, and build a trend of building products. When the building products are replaced, the system inherits and compatible with the advantages of the original building, and has the ability to remove its dross and extract its essence.

- Maintenance flexibility of buildings: in the process of building construction and subsequent maintenance, the flexible construction system can independently query the faults and conduct emergency treatment, which ensures the orderly construction and the normal operation of subsequent buildings.

- Flexible building capacity: when the building is in the process of construction, if the design of the building part changes suddenly, the building system can be well transformed and the system can operate normally.

- Extensible flexibility: when building modules need to be extended, flexible systems can readily and easily add systems to expand the original system for occasional needs.

\section{Intelligent and Flexible Construction}

\subsection{Theories and Methods}

Intelligent construction is a new construction mode, which is a full-cycle production organization mode from design to construction to later management under the influence 
of information technology and intelligent science. The main technologies include intelligent robots, $3 \mathrm{~d}$ printing, internet of things technology, virtual reality and augmented reality, etc., which are proposed to solve the problems of overstaffed management mode of traditional construction mode, poor organization and coordination, slow information transmission, slow update response, uneven construction quality, difficult maintenance in the later stage, and high dependence on physical labor. Intelligent robots can carry out high-quality construction work according to instructions. With 3D printing technology, more nonlinear houses can be built, which liberates people from heavy manual labor and changes the production mode of traditional house construction. Artificial intelligence technology has great potential in automatic design, form generation, material analysis and so on. The Internet of Things technology can accurately control the construction process by means of coding materials and components, and the real-time monitoring data of the construction model can be presented to different participants from different perspectives, which provides convenient conditions for the control of project progress, quality, safety assessment and post-maintenance [7]. These goals and flexible construction are the same, and there are many overlaps in the technology adopted. Flexible construction of the main techniques are big data, cloud computing, Internet of things, such as artificial intelligence, its starting point is the building and construction market in the future the most flexible resource use, by means of science and technology and the fine construction and construction process of changing strain, establish an effective construction organization, in order to achieve the construction engineering design, material, process, construction and the overall arrangement of funds.

\subsection{Analysis and comparison}

Intelligent construction includes the whole process of construction from design to construction to later maintenance. It can be included in the organization of production mode, the change of design mode, the design of construction tools, and the application of new materials. Its concept is more extensive, there is no fixed standard, and the research direction is also varied. Flexible construction focuses on the optimization of organizational construction forms, such as modular construction with scientific and technological means and fine construction of new materials to respond to the diversification of the construction process. Through the application of big data, cloud computing, artificial intelligence and other technologies, real-time monitoring of buildings is carried out to improve the efficiency of engineering management and the speed of response to market and demand.

\section{Conclusions}

Flexible construction system is a new concept model and a new future development trend of the construction industry. It is a very promising and strategic measure to determine the future of the construction industry. In the future development trend, intelligent construction will be combined with people, and flexible construction will be fully coordinated from the whole construction process of a series of construction industries such as scheme design, construction and subsequent maintenance.

In recent years, intelligent construction and flexible construction has become a kind of modern scientific and technological construction industry production of 
scientific philosophy and design institute in the design and construction process of automation in the advanced mode, is gradually recognized by the world, flexible construction system is based on the combination of automation technology, big data internet of things information technology, artificial intelligence technology, construction technology, and then the structure of the previous engineering construction, hydropower, technology, indoor and other independent department design, construction process, under the support of computer and various software, constitute an organic system covering the whole construction enterprise, realize the global dynamic optimization, improve the efficiency of construction, reduce the construction cost, high flexibility for the construction industry to win the competition of intelligent construction technology, flexible construction technology will point out the direction of development for the future construction industry, provides a magnificent blueprint.

\section{References}

[1] Pikorec L, Jenny D, Parascho S, Mayer H, Gramazio F and Kohler M. The brick labyrinth. In: Willmann J, Block P, Hutter M, Byrne K, Schork T C. Robotic fabrication in architecture, art and design. 2018; 2018 Sept 12-14; Zurich, Zürich. Cham (Zug): Springer; p. 489-500.

[2] Chee R, Tan W, Wei H, Amtsberg F and Dritsas S 2018. Concrete fabrication by digitally controlled injection. In: Willmann J, Block P, Hutter M, Byrne K, Schork T. c Robotic Fabrication in Architecture, Art and Design 2018; 2018 Sept 12-14; Zurich Zürich. Cham (Zug): Springer; p. 489-500.

[3] Bao Y and Li H. Artificial intelligence for civil engineering. China Civil Engineering Journal. 2019 May; 52(5): 5-15.

[4] Liu Z, Liu S, Zhao Y and Du X. Development status and future trends of intelligent construction technology. Architecture Technology. 2019 Jul; 50(7): 772-9.

[5] $\mathrm{Xu} \mathrm{W.} \mathrm{3D} \mathrm{concrete} \mathrm{printing} \mathrm{by} \mathrm{robotic} \mathrm{arms.} \mathrm{Architecture} \mathrm{Technique.} 2018$ Aug; 8: 36-7

[6] You Z, Zheng L and Feng L 2021 Basic theory and architecture of intelligent construction system Journal of Civil Engineering and Management. 2021 Apr; 38(2): 105-11.

[7] Woodhead R, Stephenson P and Morrey D. Digital construction: From point solutions to IoT ecosystem Automation in Construction. 2018 Sept; 93: 35-46. 